\title{
Current and emerging treatment options for uveal melanoma
}

This article was published in the following Dove Press journal:

Clinical Ophthalmology

2I August 2013

Number of times this article has been viewed

\author{
Patricia Rusa Pereira' \\ Alexandre Nakao \\ Odashiro $^{2}$ \\ Li-Anne Lim' \\ Cristina Miyamoto' \\ Paula L Blanco ${ }^{4}$ \\ Macanori Odashiro ${ }^{3}$ \\ Shawn Maloney' \\ Dominique F De Souza' \\ Miguel N Burnier Jr' \\ 'The Henry C Witelson Ocular \\ Pathology Laboratory, McGill \\ University, Montreal, QC, Canada; \\ ${ }^{2}$ Department of Pathology and \\ Molecular Medicine, McMaster \\ University, Hamilton, Ontario, Canada; \\ ${ }^{3}$ Federal University of Mato Grosso \\ do Sul, Campo Grande, MS, Brazil; \\ ${ }^{4}$ Department of Pathology and \\ Laboratory Medicine, University of \\ Ottawa, ON, Canada
}

Correspondence: Patricia Rusa Pereira The Henry C Witelson Ocular Pathology Laboratory, Room 216, 3775 University Street, Montreal, QC H3A 2B4, Canada Email patriciarusa@yahoo.com.br

\begin{abstract}
Uveal melanoma (UM) is the most common primary malignant intraocular tumor in adults, with a 10 -year cumulative metastatic rate of $34 \%$. The most common site of metastasis is the liver (95\%). Unfortunately, the current treatment of metastatic UM is limited by the lack of effective systemic therapy. Options for the management of the primary intraocular tumor include radical surgery as well as conservative treatments in order to preserve visual acuity. For metastatic disease, several approaches have been described with no standard method. Nevertheless, median survival after liver metastasis is poor, being around 4-6 months, with a 1 -year survival of $10 \%-15 \%$. In this review, the authors summarize current and promising new treatments for UM.
\end{abstract}

Keywords: uveal melanoma, choroidal melanoma, eye, metastasis, treatment, therapy

\section{Introduction}

Uveal melanoma (UM) is the most common primary malignant intraocular tumor in adults, presenting an age-adjusted incidence of 5.1 per million. It is a rare but sightand life-threatening malignancy. ${ }^{1}$

Modern diagnostic tools, including indirect fundoscopic examination, A and B ultrasonographic studies, optical coherence tomography, computed tomography, and magnetic resonance imaging of the globe and orbital tissues, have led to significant advances in the ability to diagnose primary UM. According to the report published by the Collaborative Ocular Melanoma Study group (COMS), the clinical diagnosis of choroidal melanomas has an accuracy of more than $99 \%{ }^{2}$ Therefore, biopsies and/or tumor resection with histopathological examination are not essential to diagnose UM. Moreover, conservative treatment of primary UM such as brachytherapy has been shown to have the same survival outcomes as surgical treatments. ${ }^{3}$ Such progress has led to new therapeutic approaches aimed towards the treatment of smaller tumors with eye conservation and useful vision. This has been accompanied by a significant decrease in patient morbidity and the number of primary enucleations performed. However, despite the treatment modality of primary UM, no decrease in the mortality rates of this tumor has been observed. In fact, the 5 -year survival rate has not changed over the last three decades $(81.6 \%),{ }^{1}$ suggesting that life expectancy is independent of successful local eye treatment. As a result, the identification of patients at high risk of metastatic disease is important, and may assist in selecting those who would benefit from adjuvant treatment. Useful prognostic factors for UM include tumor size and location, cell type, and other histopathological factors. ${ }^{4}$ More recently, genetic 
analyses have shown that UM presenting with loss of one copy of chromosome 3 (monosomy 3 ) is by far the most prognostically significant chromosomal marker in UM. Moreover, the use of gene-expression profiling seems to be a more robust prognostic method that analyzes several genes. Using gene-expression profiling, UM may be classified into three groups: those with low metastatic potential (class I), those with short-term low metastatic potential but higher mid- to long-term risk (class $\mathrm{Ib}$ ), and those with shortterm high metastatic potential (class II). ${ }^{5}$ In addition, close follow-up of these patients is paramount, as UM is well known to generate late metastasis. Recently, Daniels et $\mathrm{al}^{6}$ demonstrated that the vast majority (91\%) of large UM harbor mutually exclusive mutations in GNAQ (47\%) or GNA11 (44\%), but very rarely have the oncogenic mutations that are reported commonly in other cancers. The GNAQ and GNA11 mutations lead to activation of the mitogen-activated protein kinase pathway that consequently can be a potential target for therapy of UM that have these mutations. ${ }^{7}$

Metastasis is the leading cause of death amongst UM patients. According to the COMS study, the 5- and 10 -year cumulative metastatic rates are $25 \%$ and $34 \%$, respectively. The most common site of metastasis is the liver $(95 \%)$, followed by lung $(24 \%)$, bones $(16 \%)$, and skin $(11 \%) .{ }^{8}$ The survival rates of patients with metastatic UM is dependent upon the site of the metastasis. Median survival after liver metastasis is 4-6 months with a 1-year survival of $10 \%-15 \%$ compared with a median survival of 19-28 months with a 1-year survival of approximately $76 \%{ }^{8}$ in patients with metastasis not involving the liver. Nonetheless, Buzzacco et $\mathrm{al}^{9}$ published a series of nine long-surviving metastatic UM patients. Although not a randomized clinical trial, all nine patients underwent treatment for metastatic disease: systemic chemotherapy or biotherapy, including treatment with a tyrosine kinase inhibitor. Unfortunately, the current treatment of metastatic UM is limited by the lack of effective systemic therapy. ${ }^{10}$ In fact, some evidence shows that in most patients, cancer cells are already disseminated at the time of the diagnosis. ${ }^{11}$ The concept of tumor-cell dormancy has been discussed in UM, ${ }^{12}$ as clinically evident metastasis at the time of primary tumor diagnosis is infrequent (1\%-2\% of cases), ${ }^{13}$ and at 5 and 10 years, the cumulative metastasis rates are $25 \%$ and $34 \%$, respectively, regardless of successful treatment of the primary tumor. ${ }^{14}$ Thus, improvements in the detection of metastatic disease at the time of diagnosis of the primary tumor will assist in the selection of appropriate treatments, avoiding unnecessary local treatments and using adjuvant and adjunctive therapies. The use of whole-body positron-emission tomography/computed tomography (PET/CT) demonstrated high sensitivity and positive predictive values for detection of liver metastasis at the time of diagnosis of UM, indicating a better performance than conventional screening procedures. It is also useful for detection of extrahepatic metastases. ${ }^{15}$

\section{Treatment of the primary tumor Enucleation}

In the past, enucleation was the main treatment for the primary intraocular tumor. Still today, it is the treatment of choice when there is little chance to save vision, which is usually the case for large, advanced UM, tumors located around the optic disc, tumors presenting with extensive bleeding or retinal detachment, or vitreous hemorrhage. ${ }^{16}$ Otherwise, the UM can be managed conservatively in an attempt to save vision and the globe.

In terms of survival, several studies showed no differences in the mortality rates comparing surgical treatments and conservative treatments. ${ }^{15,16}$ In a multicenter prospective randomized trial, COMS compared enucleation and radiotherapy among similar patients with medium-sized choroidal melanomas that would be suitable for either form of treatment. With long-term follow-up, the study showed no differences in survival outcomes and little difference in quality-of-life outcomes between both methods of treatment. ${ }^{17,18}$ As a result, there has been a large shift towards eye- and vision-conserving treatments.

\section{Conservative therapy}

\section{Photocoagulation, photodynamic therapy}

Photodynamic therapy (PDT) consists of the excitation of an intravenously administered photosensitizer by a specific wavelength applied to the target region. Its tissue effects are nonthermal; generated free radicals and highly reactive singlet oxygen species inducing cell and tissue destruction through complex mechanisms that remain incompletely understood. These include cellular, vascular, and immunogenic pathways. ${ }^{17}$ The relative contribution of each pathway is thought to be dependent upon the characteristics of the photosensitizer, the treated tissue, and treatment parameters (time and dose). ${ }^{18}$ Verteporfin, a secondgeneration photosensitizer, has been shown to work primarily by inducing vascular occlusion. ${ }^{19}$ It is FDA-approved for the treatment of age-related macular degeneration, and has been used in large clinical trials with few adverse effects. ${ }^{20}$ In tumors, its effects are thought to be attributed to a combination of vascular occlusion, direct cytotoxicity, and 
activation of the immune system. ${ }^{21}$ There is some evidence however, that PDT is more effective in lightly pigmented melanomas than more densely pigmented tumors. ${ }^{22} \mathrm{~A}$ small number of in vivo choroidal melanoma animal models have achieved partial tumor remission with treatment; photosensitizing effects, including initial tumor-growth arrest and tumor necrosis, ${ }^{23}$ were demonstrated. In human studies, there is limited clinical experience. Small series using PDT with verteporfin as primary therapy have shown it to be effective in achieving complete regression. ${ }^{24}$ One further study used PDT with verteporfin as second-line therapy, demonstrating partial effect, with growth arrest achieved in two of four patients, who were subsequently able to avoid enucleation. ${ }^{22}$ However, histopathologic studies of three UM cases 1 week after treatment with PDT (with verteporfin) and bevacizumab showed viable melanoma cells with no necrosis. ${ }^{18}$ The logical conclusion is that PDT is ineffective in the treatment of these UMs, with viable UM cells seen at both short- and long-term histopathological review. This same study also found that use of PDT as a preoperative adjuvant therapy prior to biopsy eliminated bleeding at the biopsy site, an indication that may be promising in the future with further investigation. Thus, extensive clinical studies are still required to determine optimal case-selection criteria, treatment parameters, and the efficacy of PDT with verteporfin as a primary or adjuvant treatment.

\section{Transpupillary thermotherapy}

Transpupillary thermotherapy (TTT) uses an infrared diode laser to deliver low-energy phototherapy through a dilated pupil to the tumor surface. Tumor necrosis is induced by direct cell destruction. Although originally developed as an adjunct to plaque brachytherapy (BT), ${ }^{25}$ interest in TTT as a primary therapy grew based on the assumption that it may provide improved visual outcomes as compared with plaque radiotherapy. However, longer follow-up of initial studies using TTT as primary treatment for small posterior tumors revealed a high risk of local recurrence: $10 \%$ at 3 years, increasing to $33 \%$ at 10 years. ${ }^{26-28}$ Similarly, a literature review of ten related series and pooled data of 602 tumors and 100 recurrences revealed a weighted mean tumor recurrence of $17 \%$ at a median follow-up of 37 months, further demonstrating TTT as primary therapy to be ineffective. ${ }^{29}$ In addition, although only shown in a small retrospective case-matched study of 36 patients, ${ }^{27}$ there was no significant difference in visual acuity outcomes between patients treated with TTT compared with plaque BT. Complications of primary TTT include branch retinal artery and vein occlusions, epiretinal membranes, cystoid macular edema, and optic disk swelling. ${ }^{28}$ Thus, current evidence limits TTT to use as an adjunct to plaque BT. Several studies of combined TTT and BT have been published to date, ${ }^{25,30,31}$ however, they consist of varying treatment regimes with regard to the timing of TTT and the use of various isotopes. Most recently, the largest retrospective comparative study to date of 133 patients treated either with BT + TTT $(n=63)$ or with BT alone $(n=70)$ revealed there to be significant benefits from simultaneous TTT + BT. Such combined treatment provided higher local control $(P=0.036)$, eye retention $(P<0.024)$, and recurrence-free survival $(P<0.034)$, with similar rates of metastases and overall survival ${ }^{32}$ compared with BT alone. Its role in the management of foveal subretinal fluid associated with small UM also appears promising. ${ }^{33}$

\section{lonizing radiation}

Radiotherapy acts by inducing DNA damage, resulting in tumor-cell death and proliferation arrest of remaining surviving cells. ${ }^{34}$ For the treatment of UM, radiation may be delivered using a variety of different methods, including plaque BT, charged-particle therapy (CPT), and gamma-knife and stereotactic delivery systems.

\section{Plaque brachytherapy}

Following the results of the medium-sized choroidal melanoma COMS trial, ${ }^{35}$ the use of reusable gold-shielded episcleral plaques ${ }^{36}$ and the COMS-standard BT protocol have become widely available at multiple centers. Many different radioactive isotopes have been used in ocular BT, their specific half-life and tissue penetration properties making them useful in the treatment of UM tumors of different sizes. Ruthenium $106\left({ }^{106} \mathrm{Ru}\right)$ emits beta radiation, which has a limited penetration in contrast to iodine 125 $\left({ }^{125} \mathrm{I}\right)$, which emits gamma rays that penetrate more deeply and can be used to treat larger tumors adequately of up to $10 \mathrm{~mm}$ thick. Similar tumor-control rates have been reported with both isotopes, ${ }^{37}$ although a significantly greater risk of tumor recurrence has been reported for tumors treated with ${ }^{106} \mathrm{Ru}$ compared with either ${ }^{125} \mathrm{I}$ or proton-beam radiotherapy in a series of 597 patients. ${ }^{38}$ Unfortunately, greater radiation penetrance is also associated with an increased risk of damage to surrounding ocular tissues. The most common radiation side effects include optic neuropathy, maculopathy, cataract, and neovascular glaucoma, numerous studies of which have shown these to be dose-dependent. ${ }^{39,40}$ BT does, however, provide excellent tumor control, with local control rates exceeding $90 \% .{ }^{41} \mathrm{~A}$ single-center study of 458 patients 
with predominantly medium-sized choroidal melanomas treated with ${ }^{108} \mathrm{Ru}$ brachytherapy reported actuarial rates of tumor recurrence to be $1 \%, 2 \%$, and $3 \%$ at 2,5 , and 7 years, respectively. ${ }^{42}$ Visual outcomes are dependent upon tumor location and thickness, with one large study reporting an actuarial rate of $55 \%$ for conservation of vision $20 / 40$ or better at 9 years. ${ }^{43}$ Correct plaque positioning is essential for good clinical outcomes. ${ }^{43}$ For the treatment of juxtapapillary tumors, good local control can still be achieved with plaque BT. ${ }^{44}$ The use of eccentric plaque placement has been shown to provide good local control for tumors within $5 \mathrm{~mm}$ of the optic disk or fovea. ${ }^{45}$ Slotted plaques incorporate the optic nerve into the plaque, allowing the entire tumor and a $2 \mathrm{~mm}$-free margin to be encompassed by the plaque. A recent study of 24 consecutive patients with juxtapapillary UM reported a $100 \%$ local control rate at a mean follow-up of 23 months. $^{46}$

\section{Charged-particle therapy}

CPT may be delivered using protons, helium, or carbon ions. In contrast to conventional radiation therapy, CPT allows for more focused radiation treatment, collimated charged-particle beams peaking at the desired tissue depth and stopping thereafter (Bragg peak). This allows for the delivery of large radiation doses for adequate tumor treatment with no exit dose beyond the tumor and theoretical sparing of surrounding structures. A recent systematic review and meta-analysis by Wang et $\mathrm{al}^{47}$ reviewed the current evidence for the efficacy of CPT in the treatment of UM. Of the 27 studies that met inclusion criteria, there was only one randomized control trial ${ }^{48}$ of 184 treatment-naïve patients, comparing helium ion therapy $(n=86)$ to ${ }^{125} \mathrm{I}$ BT $(\mathrm{n}=98)$, the rest being observational studies. From the pooled data for treatment-naïve patients (26 studies), there was a strong and significant reduction of local tumor recurrence associated with CPT (odds ratio [OR] 0.22, 95\% confidence interval [CI] 0.21-0.23). Between the two treatments, CPT and ${ }^{125} \mathrm{I} \mathrm{BT}$, there was no significant reduction in mortality (OR $0.13,95 \%$ CI 0.01-1.63), or significant difference in risk of subsequent enucleation (OR 0.53, 95\% CI 0.23-1.18). The five most commonly reported adverse effects associated with CPT were glaucoma, radiation retinopathy, cataract formation, optic neuropathy, and enucleation due to complications. When compared with patients treated with ${ }^{125} \mathrm{I}$ BT in the COMS trial, ${ }^{49-51}$ there were significantly lower rates of radiation retinopathy and cataract formation among patients treated with CPT ( 0.28 vs 0.42 and 0.23 vs 0.68 , respectively). Ten studies reported visual acuity outcomes; however, heterogeneity between studies limited the statistical pooling of results. One single-institution study of 349 patients treated with CPT (proton-beam radiotherapy) $^{52}$ reported a conservation rate of good visual acuity of 20/40 or better of $63.5 \%$ (95\% CI $55.9 \%-71.1 \%)$ at 2 years, $44.8 \%(95 \%$ CI $35.3 \%-54.4 \%)$ at 5 years, and $32.2 \%(95 \%$ CI $21.1 \%-43.2 \%)$ at 8 years. Loss of vision was strongly correlated with posterior tumor extension $(P=0.001$; risk ratio $1.58,95 \%$ CI $1.22-2.05)$, which has also been reported by other groups. ${ }^{53}$ Unfortunately, CPT often also administers radiation to the anterior segment in order to reach posterior UM tumors. The percentage of lens and anterior chamber involvement in the treatment field has been shown to correlate strongly with the development of neovascular glaucoma. This may be decreased with a two-field approach and sparing of the anterior chamber. ${ }^{54}$ CPT techniques that aim to reduce the direct complications of radiation include the use of notched beams, adjunctive TTT or phototherapy, and treatment through a closed eyelid, all of which are still under investigation. ${ }^{52}$ Data from the UM registry ${ }^{53}$ suggest that patients with larger tumors and tumors near the optic disk and fovea are preferentially referred to centers that offer CPT.

\section{Stereotactic delivery systems}

Stereotactic radiation therapy 55 and gamma-knife radiosurgery ${ }^{56}$ also provide good local control, with survival rates comparable with other treatments. However, the radiation dose distribution is not as precise as with PCT. Furthermore, the current experience is limited, with relatively short follow-up periods and a lack of comparison with brachytherapy and CPT.

\section{Local resection}

Local resection of a choroidal melanoma may be an alternative conservative treatment option that allows for removal of the tumor with retention of the eye. Furthermore, it allows for histopathological and cytogenetic analysis. The approach may be transretinal (endoresection), which is more suitable for posterior tumors, or transscleral (exoresection), which typically requires hypotensive anesthetic conditions to reduce the risk of hemorrhage. To prevent tumor recurrence, adjunctive plaque radiotherapy is recommended for exoresection, ${ }^{57}$ whilst the need for neoadjuvant radiation prior to endoresection remains controversial. ${ }^{58}$ Unfortunately, there is no prospective comparative data available addressing this issue or that of morbidity and risk for metastasis. Major complications of both approaches include retinal detachment, ${ }^{57}$ vitreous hemorrhage, cataract, and elevated intraocular 
pressure, ${ }^{59}$ some of which may occur unpredictably. Identified risk factors for severe visual loss (hand movements or worse) include posterior tumor extension to within 1 disk diameter of the optic disk and/or fovea $(P=0.009) .{ }^{60}$

\section{Adjuvant treatments}

Adjuvant therapy (AT) may consist of radiotherapy or systemic therapy, such as chemotherapy, immunotherapy, hormone therapy, biological therapy, or target therapy. Although this treatment modality is well established in some tumors, there are few studies of AT in UM.

Lane et a ${ }^{61}$ treated 121 high-risk UM patients with adjuvant interferon alfa-2a after radiation or enucleation from 1995 to 1999 . They defined high-risk patients as: age $\geq 56$ years, largest tumor dimension $\geq 15 \mathrm{~mm}$, ciliary body involvement, or extrascleral tumor extension. The therapy was applied as 3 million International Units subcutaneously three times per week over a 2-year course. This AT, however, had no significant influence on melanoma-related mortality (rate ratio $1.02,95 \%$ CI $0.68-1.5 ; P=0.91)$.

Gomer ${ }^{17}$ treated 22 high-risk UM patients with AT intra-arterial hepatic fotemustine. Planned treatment duration was 6 months, starting with 4-weekly doses of $100 \mathrm{mg} / \mathrm{m}$, and after a 5-week rest this was repeated every 3 weeks. The 5-year survival rate of the experimental group was $75 \%$, compared to $56 \%$ of a matched-control group. Their data suggested a survival benefit, although it was not statistically significant.

Some studies have been done applying AT in local eye treatment. De Potter and Jamart ${ }^{62}$ applied indocyanine green in 30 patients with choroidal melanoma before treatment with TTT. They found no difference in the regression pattern compared to 30 patients treated only with TTT. Nonetheless, in vitro studies showed that UM cells treated with amfenac, a cyclooxygenase- 2 inhibitor, become more radiosensitive and may decrease tumor recurrence and radiation-induced complications while broadening the indications for radiotherapy in UM tumors. ${ }^{63}$

In conclusion, there are very few studies of AT for UM. More studies with different promising systemic therapies and combination treatments are needed. The low incidence rate of this tumor may contribute to the difficulty in establishing clinical trials.

\section{Locoregional treatments of liver metastasis}

The liver is usually the first site of metastasis of UM, and is the only single organ involved in $60 \%-80 \%$ of these patients. ${ }^{64}$ The median survival of patients with liver metastasis is $2-7$ months despite aggressive therapy, ${ }^{22}$ and the clinical course of these patients is highly dependent on disease progression in the liver. ${ }^{8}$ Different approaches have been used to treat metastatic liver disease in order to improve survival, including surgery, hepatic intra-arterial (HIA) chemotherapy, chemoembolization, immunoembolization, and isolated hepatic perfusion (IHP). However, there is no standard treatment for liver metastasis.

\section{Resection of metastatic nodules}

It is well known that in liver-limited metastasis of colon cancer, hepatectomy is the best therapeutic strategy in terms of prognosis ${ }^{65}$ This is true in other types of cancer as well. Mariani et al ${ }^{66}$ showed that complete resection of UM liver metastasis, either by single or multiple resections, improves the survival of these patients. The presence of "miliary" metastases is a contraindication for major liver resection. They found that the time to liver metastasis ( $>24$ months), comprehensiveness of liver resection (microscopically complete), number of liver metastases resected $(\leq 4)$, and absence of miliary disease were correlated with overall prolonged survival. Frenkel et a ${ }^{67}$ also demonstrated that resection of the metastatic liver nodule improves survival. Survival rates of patients who had one to five metastatic nodules were 3.1 times longer than those who had more than six metastatic nodules, and patients with complete resection of the hepatic metastasis with clean histological margins had 1.9-times longer survival than those with residual disease. Yet the literature shows many isolated cases of long-surviving patients following resection of liver metastasis. ${ }^{68}$ Therefore, especially in limited UM liver metastasis, surgical resection seems to improve the survival of these patients. However, only a limited number of patients are eligible for surgical treatment, as most of them present with multiple liver metastases involving both liver lobes. ${ }^{69}$ In some reports, the median survival of operated patients was 23 months after surgery, ${ }^{67}$ and in others it was 14 months, but increased to 27 months when complete resection was possible. ${ }^{66}$

\section{Hepatic intra-arterial chemotherapy}

HIA chemotherapy is a modality of treatment in which the drug is delivered via an indwelling hepatic artery catheter placed by the surgeon. It allows for maximum local drug exposure with rapid systemic clearance. ${ }^{70}$

Several drugs have been tested for use as HIA chemotherapy as a treatment for UM liver metastases. Fotemustine was demonstrated to be well tolerated and shown to improve outcomes compared with a systemic treatment 
approach. It showed an overall response rate of $36 \%$, with a median overall survival of 15 months and a 2-year survival rate of $29 \% .{ }^{31}$ Melphalan has also been tested with good survival times and acceptable major complication rates. ${ }^{32}$ In this study, patients with as many as nine liver metastatic nodules but without extrahepatic metastasis at the beginning of therapy had longer survival rates with treatment. The combination of cisplatin, vinblastine, and dacarbazine via HIA chemotherapy was tested in ten patients with metastatic UM, with similar efficacy to other HIA chemotherapy regimes. ${ }^{71}$

\section{Intra-arterial hepatic chemoembolization}

Hepatic transarterial chemoembolization (TACE) combines artery embolization with the infusion of chemotherapy drugs. It has been shown to be effective in the treatment of hepatocellular carcinoma ${ }^{72}$ as well as metastatic disease to the liver. ${ }^{73}$

Huppert et $\mathrm{al}^{74}$ treated 14 patients with UM liver metastasis with cisplatin and/or carboplatin TACE. They found that this procedure is well tolerated and may prolong survival in patients with limited tumor extension (less than $25 \%$ of liver volume). BCNU (1,3-bis[2-chloroethyl]-1nitrosourea) by TACE was tested in 24 patients with hepatic UM metastasis. Eighteen of these 24 patients experienced regression or stabilization of hepatic metastases with longer survival rates. However, 13 of the 18 patients who achieved response or stable disease subsequently developed progression of extrahepatic metastases with control of hepatic metastases. ${ }^{37}$ Carrasco et a ${ }^{75}$ successfully treated two patients with liver metastasis by TACE with a combination of cisplatin and polyvinyl sponge. However, the same results were not observed by other authors in larger series. ${ }^{39,40}$ Nonetheless, TACE has been considered a more effective treatment compared to systemic conventional chemotherapy, especially using BCNU. ${ }^{42}$

\section{Immunoembolization}

Immunoembolization is a technique where embolization of the hepatic artery is performed with immune-stimulating agents such as granulocyte-macrophage colony-stimulating factor (GM-CSF). GM-CSF is a glycoprotein secreted by $\mathrm{T}$ cells that stimulates immune cells, such as macrophages and dendritic cells. Immunoembolization provides attraction and stimulation of these immune cells in liver tumors that may eliminate residual tumor cells. It seems to be a safe and effective salvage therapy for limited UM liver metastases. ${ }^{76}$ Sato et al studied 34 patients treated with immunoembolization with promising results. They found an overall median survival of 14.4 months, and 1- and 2-year survival rates of $62 \%$ and $26 \%$, respectively. ${ }^{77}$ The same group showed that patients treated by immunoembolization had a better overall survival than patients treated by TACE with BCNU (20.4 vs 9.8 months). Prognostic factors associated with longer progression-free survival were patients that received a high dose of immunoembolization, age $<60$ years, and regression of hepatic metastases. ${ }^{78}$

\section{Isolated hepatic perfusion}

IHP requires a complex and expensive surgical procedure with considerable morbidity and mortality, which is largely related to veno-occlusive disease and hepatotoxicity. ${ }^{79}$ However, it is does allow for a higher concentration delivery of chemotherapy to the liver, whilst avoiding severe undesirable systemic effects. Most studies with IHP have been done in the setting of metastatic colorectal cancer to the liver, with only a few studies looking at its role in the treatment of liver metastases secondary to UM.

\section{Stereotactic liver radiotherapy}

Oligometastatic patients (metastases limited in number and location) may benefit from stereotactic liver radiotherapy in diverse types of cancer. Primarily, it was designed for patients who are not medically fit for surgery, or those who are technically unresectable, to be treated with a few fractions of high doses of radiation to each lesion. ${ }^{80}$ This is an emerging area in radiation oncology, and the efficacy in UM metastatic patients should be studied. A study showed that a dose of 60 Gy in five fractions seems to give an excellent level of local control and can be safely delivered to selected patients with hepatic metastases as long as the critical liver volume is respected. ${ }^{81}$

\section{Systemic therapies \\ Conventional chemotherapy}

Systemic chemotherapy is reserved for metastatic UM. Various chemotherapeutic agents have been investigated; however, at present there are no standard systemic therapies for this stage of the disease.

Initially, treatments applied to cutaneous melanoma were tested in UM, despite their different biology. Although promising results with a combination of bleomycin, vincristine, lomustine, and dacarbazine ${ }^{50}$ with interferon- $\alpha 2^{82}$ were obtained in the beginning, the Eastern Cooperative Oncology Group (EORTC) proved that these results were eventually overoptimistic in a larger number of patients. 
Of the 26 patients enrolled in the study, none achieved an objective response, two (8.3\%) remained stable, 20 showed progression, and two $(8.3 \%)$ were not able to be evaluated. The median progression-free survival was 1.9 months, and overall survival was 10.6 months. ${ }^{83,84}$ Furthermore, data also from the EORTC suggest that liver metastasis from UM has a lower response rate (10\%) than metastasis from cutaneous melanoma (33\%) when patients are treated with the "Darmouth combination" (dacarbazine, carmustine, cisplatin, and tamoxifen). ${ }^{85}$

To date, no randomized trial has reported the superiority of any therapeutic strategy to best support care, as in cutaneous melanoma. A variety of cytotoxic agents have been investigated, such as dacarbazine, treosulfan, temozolomide, fotemustine, cisplatin, and combination therapies as previously mentioned, such as bleomycin, vincristine, lomustine, and dacarbazine (BOLD). ${ }^{86}$ The response rate to systemic chemotherapy ranges from $0 \%$ to $15 \%$, with no evidence that it extends survival in patients with metastatic UM. The survival remains between 2 and 7 months, with only $15 \%$ of patients alive at 1 year. As previously stated, higher response rates and median survival have been reported with chemotherapy administration directly into the hepatic artery in a highly selected group of patients. ${ }^{87}$

A tentative trial administering treosulfan, a prodrug used for many years in the treatment of ovarian cancer, failed to show any clinical activity in a Phase II study of patients with metastatic UM. ${ }^{10}$

A randomized Phase II trial tested gemcitabine and treosulfan against treosulfan alone, based on preclinical evidence of synergy. The clinical benefit-complete response, partial response, or stable disease - favored the combination at $33 \%$ versus $12.5 \%$ for treosulfan alone. However, the response rate in the combination was only $4 \%$ and the progression free-survival was 3 months. ${ }^{86,88}$

Another Phase II study with cisplatin, gemcitabine, and treosulfan involving 48 subjects reported no objective responses in 17 evaluable patients and only seven patients with stable disease. The median progression free-survival and overall survival were 3 and 7.7 months, respectively. ${ }^{10,89}$ In one more Phase II study, 15 patients received treosulfan and dacarbazine at $8 \mathrm{mg} / \mathrm{mq}$ and $850 \mathrm{mg} / \mathrm{mq}$, respectively, every 21 days. Although well tolerated, this type of treatment was not associated with an increment of objective responses. ${ }^{10,90}$

There is no optimal chemotherapy for metastatic UM; however, the treatments evaluated in a study by Pons et al have at least shown some activity in this disease, with no other active schedules available. These regimes included dacarbazine, temozolomide with or without interferon, fotemustine, carboplatin/dacarbazine/interferon- $\alpha /$ interleukin 2 (IL-2) ${ }^{87}$ Dacarbazine is still the most recognized treatment in combination or in monotherapy in melanoma. However, uncertainty remains with regard to the level of activity in UM as opposed to cutaneous melanoma ${ }^{87}$ From a retrospective review of cases treated at the MD Anderson Cancer Center, Bedikian et al concluded that standard systemic chemotherapy was inactive, with a response rate as low as $1 \% .^{87,91}$ In contrast however, Flaherty et al reported that both uveal and cutaneous melanomas have similar response rates, according to their experience with patients entered into seven consecutive Phase II trials carried out by the Southwest Oncology Group..$^{87,92}$ Temozolomide in monotherapy has been shown to be ineffective for this disease, but the experience of combining this drug with interferon- $\alpha 2 b$ in UM has never been reported. ${ }^{87}$ The two Phase II trials with better survival in UM, however, are the only ones that combine interferon- $\alpha$ with bleomycin + vincristine + lomustine + dacarbazine or fotemustine. ${ }^{87,93}$

Despite poor results in a Phase II trial, ${ }^{93}$ fotemustine has one of the best response rates and overall survival data compared with other schedules, and has been adopted as a standard for treatment in patients with metastatic UM by European groups. ${ }^{87}$

New chemotherapeutic agents tested in uveal melanoma include docosahexaenoic acid-paclitaxel and vincristine sulfate liposomes. Homsi et $\mathrm{al}^{94}$ conducted a Phase II study to evaluate docosahexaenoic acid-paclitaxel, a covalent conjugate of paclitaxel and docosahexaenoic acid, for safety and response rate. Docosahexaenoic acid-paclitaxel was administered at $500 \mathrm{mg} / \mathrm{mq} /$ week for 5 consecutive weeks in 22 patients. One chemonaïve patient achieved partial response for 5 months, and seven patients (32\%) had stable disease for 3 months. The median overall survival was 9.8 months. ${ }^{10}$

Vincristine sulfate liposomal infusion is a sphingomyelin/ cholesterol liposome encapsulated formulation of vincristine that results in extended drug circulation time and anticancer activity. In a pilot Phase I study ${ }^{95}$ including cutaneous and UM patients, the disease-control rate was $31 \%$, with one complete response (UM with lung metastases) and two partial responses (previously untreated cutaneous melanoma). The median survival was 9.6 months. A Phase II trial is enrolling patients in order to elucidate the safety and efficacy of vincristine sulfate liposomal infusion in metastatic UM patients. ${ }^{10}$

The EORTC Melanoma Group is recruiting patients with unresectable liver metastases from UM for the first 
randomized Phase III trial for this stage of the disease. This study will compare treatment with intravenous fotemustine versus intra-arterial hepatic perfusion. The final results will show how these therapies influence overall survival. ${ }^{10,87}$ A summary of some studies about chemotherapy and UM is shown in Table 1.

\section{Target therapies}

Target therapy (TTh) is a relatively new modality of cancer treatment and one of the most studied fields in cancer today. It applies drugs that block specific pathways to the growth and evolution of cancer cells by interfering with specific molecules involved in tumor proliferation and progression. It has being studied for use alone, in combination with conventional chemotherapy, or in combination with other TThs. TTh may be promising in UM, as metastatic UM has a poor response to conventional chemotherapy. ${ }^{12}$ In fact, there are several clinical trials currently testing different types of TTh in UM.

C-kit (CD117, stem cell-factor receptor) is a transmembrane receptor with tyrosine kinase activity that plays a role in many vital cellular processes, including differentiation, proliferation, and programmed cell death. It was shown to be overexpressed in many human cancers, ${ }^{96}$ and its clinical interest became apparent with the discovery of imatinib mesylate (IM), a tyrosine kinase inhibitor that blocks c-kit and its molecular signaling pathway, as well as two other tyrosine kinase receptors: bcr-abl and platelet-derived growth-factor receptor. IM is indicated for the treatment of patients with Philadelphia chromosome-positive chronic myeloid leukemia and patients with unresectable and/or metastatic malignant gastrointestinal stromal tumors. C-kit was shown to be overexpressed in UM primary tumors as well as in metastatic lesions. ${ }^{47-49}$ Some clinical trials using IM as monotherapy in advanced UM patients showed there to be no clinical improvement. ${ }^{50,51}$ However, it was demonstrated that in vitro, IM effectively decreases the invasion capability of UM cells and also affects, although in a less successful way, the proliferation of UM cells. ${ }^{97}$ Therefore, new studies are necessary in order to address the effect of IM as AT in the treatment of primary intraocular UM tumors, especially in high-risk patients. A recent in vivo study from our laboratory

Table I Chemotherapy for metastatic uveal melanoma $8,74,77,80,81,85,86$

\begin{tabular}{|c|c|c|c|c|c|c|c|c|}
\hline \multirow[t]{2}{*}{ Lead author } & \multirow[t]{2}{*}{ Year } & \multirow{2}{*}{$\begin{array}{l}\text { Number of } \\
\text { patients }\end{array}$} & \multirow[t]{2}{*}{ Chemotherapy } & \multirow{2}{*}{$\begin{array}{l}\text { Median survival } \\
\text { (months) }\end{array}$} & \multicolumn{3}{|c|}{ Response } & \multirow[t]{2}{*}{ Type of study } \\
\hline & & & & & CR & PR & $\%$ & \\
\hline Einhorn et al ${ }^{1 / 2}$ & 1974 & 25 & Various & 8.5 & 0 & 4 & 16 & Retrospective \\
\hline Rajpal et al ${ }^{110}$ & 1983 & $7^{*}$ & Various & 4.5 & & & & Retrospective \\
\hline Gragoudas et $\mathrm{al}^{1 / 3}$ & 1991 & $61 *$ & Various & 3.8 & & & & Review \\
\hline Pyrhonen et al ${ }^{1 / 4}$ & 1992 & 4 & BOLD + interferon- $\alpha$ & - & 0 & 2 & 50 & Phase I \\
\hline Kath et $\mathrm{al}^{115}$ & 1993 & $14^{*}$ & Various & 9 & 0 & 0 & 0 & Retrospective \\
\hline Nathan et al ${ }^{116}$ & 1994 & 16 & Dartmouth & - & 0 & I & 6 & Prospective uncontrolled \\
\hline Bedikian et $\mathrm{al}^{91}$ & 1995 & 143 & Various & 6 & 0 & I & $<1$ & Retrospective \\
\hline Atzpodien et $\mathrm{al}^{117}$ & 1995 & $7^{\#}$ & $\begin{array}{l}\text { Dacarbazine + carboplatin or } \\
\text { Dartmouth + interferon- } \alpha 2\end{array}$ & & - & - & - & Phase II \\
\hline Albert et $\mathrm{al}^{85}$ & 1996 & 51 & Various & 4.5 & 0 & 0 & 0 & Review \\
\hline Proebstle et $\mathrm{al}^{1 / 8}$ & 1996 & 8 & $\begin{array}{l}\text { Dacarbazine }+ \text { cisplatin }+ \\
\text { interferon- } \alpha 2 b\end{array}$ & - & 0 & I & 12 & Phase I \\
\hline Nathan et al'19 & 1997 & 20 & BOLD + interferon- $\alpha 2 b$ & - & 0 & 4 & 20 & Prospective uncontrolled \\
\hline Flaherty et $\mathrm{al}^{92}$ & 1998 & 64 & Dacarbazine or cisplatin & 5 & I & 5 & 9 & Prospective uncontrolled \\
\hline Pyrhonen et al ${ }^{120}$ & 2002 & 20 & BOLD + interferon- $\alpha$ & 12 & 0 & 3 & 15 & Prospective uncontrolled \\
\hline Kivelä et $\mathrm{al}^{84}$ & 2003 & 24 & BOLD + interferon- $\alpha 2 b$ & II & 0 & 0 & 0 & Prospective uncontrolled \\
\hline Pfohler et al ${ }^{121}$ & 2003 & 14 & Treosulfan + gemcitabine & 14 & I & 3 & 29 & Prospective uncontrolled \\
\hline Bedikian et $\mathrm{al}^{122}$ & 2003 & 14 & Temozolomide & 7 & 0 & 0 & 0 & Prospective uncontrolled \\
\hline Schmittel et $\mathrm{al}^{89}$ & 2005 & 33 & Gemcitabine + treosulfan & 9 & 0 & I & 5 & Phase I \\
\hline Schmittel et $\mathrm{al}^{86}$ & 2006 & 19 & $\begin{array}{l}\text { Gemcitabine }+ \text { treosulfan }+ \\
\text { cisplatin }\end{array}$ & 7.7 & 0 & I & 0 & Phase II \\
\hline O’Neill et al ${ }^{90}$ & 2006 & 14 & Dacarbazine + treosulfan & 7.2 & I & 3 & 29 & Phase II \\
\hline Bedikian et $\mathrm{al}^{95}$ & 2008 & 4 & Vincristine sulfate liposomal & 9.6 & I & 2 & 31 & Pilot \\
\hline Homsi et al $^{94}$ & 2010 & 22 & Docosahexaenoic acid-paclitaxel & 9.8 & 5 & I & 5 & Phase II \\
\hline
\end{tabular}

Notes: *Treated with chemotherapy alone; "not segregated from cutaneous melanoma. Dartmouth refers to a regimen of dacarbazine + carmustine + cisplatin + tamoxifen.

Abbreviations: CR, complete response; PR, partial response; BOLD, bleomycin, vincristine, lomustine, and dacarbazine. 
testing IM in a rabbit animal model offered new insights into the utility of this drug in UM..$^{53}$ IM treatment resulted in fewer and smaller intraocular tumors, and fewer metastases.

Nuclear factor-kappa B (NFkB) is a factor that regulates the transcription of genes involved in apoptosis, proliferation, angiogenesis, immune response, cell invasion, and cell adhesion. It is known to be involved in the development of different malignancies, including hepatocarcinoma and retinoblastoma. Jampol et $\mathrm{al}^{49}$ demonstrated that $\mathrm{NF \kappa B}$ is expressed by primary UM and its liver metastases, NFKB inhibitors reducing metastatic cell proliferation. Combination of bortezomib, an NFאB inhibitor, and conventional therapy is currently being studied. A clinical trial treating metastatic UM patients with bortezomib, paclitaxel, and carboplatin is ongoing at the Mayo Clinic, Rochester, MN, USA (NCT00288041).

Heat shock protein 90 (Hsp90) is a central chaperone protein involved in the pathway of several client proteins, such as growth factor receptors, signaling kinases (Akt and Raf-1), and cell-cycle regulators (cyclin-dependent kinase 4), which regulate signal transduction, cell-cycle control, and antiapoptotic mechanisms. Hsp90 is overexpressed in several malignancies, and may represent a promising therapeutic strategy. In vitro studies showed that most UMs express this protein, inhibition of Hsp90 by $17-N$-allylamino17-demethoxygeldanamycin significantly decreasing the migratory and invasive capabilities of UM cells. ${ }^{98}$ In fact, a clinical trial (NTC1200238) with metastatic UM treated by STA-9090 (ganetespib), an Hsp90 inhibitor, is currently being conducted. No results have been published so far.

As mentioned, about $80 \%{ }^{99}$ to $91 \%$ of large UMs have mutations in the GNAQ or GNA11 genes, ${ }^{6}$ and these mutations are associated to activation of the mitogen-activated protein kinase pathway. ${ }^{7}$ A Phase I clinical trial testing trametinib, a selective inhibitor of the Ras-Raf mitogen-activated protein kinase (MEK) pathway, was conducted in 81 skin melanoma and $16 \mathrm{UM}$ patients. The results showed substantial clinical activity of trametinib in melanoma, suggesting that MEK is a valid therapeutic target. Differences in response rates according to mutations indicate the importance of mutational analyses in the future. ${ }^{55}$ Another clinical trial testing a selective MEK pathway for metastatic UM patients is being conducted (NCT01143402). Patients are treated either with temozolomide or selumetinib. Selumetinib is a potent and selective inhibitor of the MEK pathway. ${ }^{56}$ Somatic activating mutations in the RAS/RAF/MEK/ERK signaling pathway are frequent in cutaneous melanomas, with $50 \%-70 \%$ of them harboring $B R A F$ mutations. $B R A F$ cutaneous melanomas are highly sensitive to MEK inhibition. However, in UM, $B R A F$ mutations are rare. Bechrakis et $\mathrm{al}^{53}$ investigated the impact of B-Raf and MEK inhibition on UM cell lines using small-molecule inhibitors, either as monotherapy or in combination with each other and the Akt inhibitor. They found that the $B R A F$-mutant UM cells behave similarly to their cutaneous counterparts, with high sensitivity to inhibition of either B-Raf or MEK that can be further enhanced by concurrent Akt inhibition. However, non-BRAF-mutant UM cells are less sensitive to MEK inhibition (but can be further sensitized by concurrent Akt inhibition) and are completely resistant to B-Raf inhibition (even in the presence of the Akt inhibitor). It is well known that UM and cutaneous melanoma have different genetic mutations, although both have high mortality rates. Therefore, the rationale is to consider them as distinct entities, and the ideal is to study them individually. Nonetheless, there is a Phase III study (NCT01245062) assessing the efficacy of an MEK inhibitor (trametinib) in progression-free survival and overall survival compared with chemotherapy in patients with BRAFV600E/K mutant advanced or metastatic cutaneous melanoma. Preliminary results showed a significant difference between the groups, with progression-free survival in patients treated by the MEK inhibitor and chemotherapy of 4.8 months vs 1.4 months, respectively.

Sunitinib is an oral, multitargeted receptor tyrosine kinase that inhibits receptors for platelet-derived growth factor, vascular endothelial growth factor (VEGF), c-Kit, RET, CSF-1R, and Flt3. It is FDA-approved for the treatment of renal cell carcinoma and imatinib-resistant gastrointestinal stromal tumor. ${ }^{100}$ Studies have also looked at the role of VEGF in UM metastasis and progression. A Phase II clinical trial (NCT00489944) is also currently in progress, looking at the side effects and synergistic effects of combination treatment with sunitinib, tamoxifen, and cisplatin in the treatment of high-risk ocular melanoma patients.

Tumor angiogenesis is the formation of tumor-associated vasculature, which is essential for tumor growth, progression, and metastasis. VEGF is one of the major cytokines that influence angiogenesis, described in several malignancies, including UM. ${ }^{61}$ Animal-model studies have shown that hepatic micrometastatic UM secretes VEGF, the number and location of the micrometastases correlating with serum VEGF levels. ${ }^{101}$ Barak et al ${ }^{102}$ demonstrated that serum VEGF increased significantly after metastatic development in UM patients and that it could serve as a biomarker for metastatic UM. Preclinical studies demonstrated that bevacizumab, a monoclonal anti-VEGF antibody, suppressed 
in vitro growth and in vivo hepatic micrometastasis of ocular melanoma cells. ${ }^{63}$ However, some in vitro studies have found paradoxical results, with an increase in tumor growth after bevacizumab treatment. A group from France is currently studying the combination of bevacizumab and temozolomide in UM patients (NCT01217398).

Oblimersen is an antisense oligodeoxyribonucleotide that targets bcl-2. Bcl-2 is an apoptosis regulator protein related to several types of cancer. Some studies suggest that this protein may play a role in $\mathrm{UM},{ }^{62,64}$ being responsible for the general lack of susceptibility to apoptosis in this tumor. ${ }^{103}$ A clinical trial testing the combination of oblimersen, carboplatin, and paclitaxel is ongoing in Houston, TX, USA (NCT01200342).

Most of the studies testing new therapies in metastatic UM are Phase I/II clinical trials. The diversity of pathways involved in the metastatic pathway of UM makes it difficult to find a specific effective drug for this highly lethal tumor. In our opinion, multiple TTh drugs inhibiting different pathways in combination with conventional chemotherapy are necessary to test. In fact, in 2008, Augsburger et al, ${ }^{104}$ in a review of the current literature concerning treatments in metastatic UM, showed that there was little evidence of survival improvement among different treatment modalities for these patients.

\section{Immunotherapy}

The application of cytokines to induce or promote the generation of an effective antitumor immune response is an attractive approach in cancer immunotherapy. ${ }^{105}$ As UM arises in an immune-privileged site, the eye, it could be responsive to T-cell-based immunotherapy. Most of the immunotherapy treatments tested in UM are extensions from previous experiences with cutaneous melanoma, but the genetic differences between these two tumors indicate that an immunotherapy specifically for UM should be developed. ${ }^{106}$ Ipilimumab is a fully human anticytotoxic T-lymphocyte antigen-4 monoclonal antibody that seems to improve overall survival in patients with advanced cutaneous melanoma. Danielli et al ${ }^{107}$ performed a multicenter study with ipilimumab of 13 pretreated patients with metastatic UM in six different European institutions. No objective responses were observed, but two patients achieved stable disease, a third achieving stable disease after initial disease progression.

IL-2 is an FDA-approved immunostimulatory cytokine for stage 4 cutaneous melanoma, with an overall response rate of $20 \%$ and complete response rate of $5 \%-10 \%{ }^{70}$ However, no clinical trials of IL-2 in metastatic UM have been performed. Soni et al ${ }^{108}$ reported an 8-year-old girl with UM metastatic to liver and pancreas who was treated with highdose bolus IL-2 and thalidomide. The patient experienced minimal toxicity and had stable disease for 23 months. From a series of nine cases of long-surviving metastatic UM patients, two received IL-2 and one received IL-2 plus programmed death (PD)-1 monoclonal antibody vaccine. ${ }^{9}$

The combination of BOLD with interferon- $\alpha$ showed objective response rates of approximately $20 \%, 82$ however, further studies did not confirm this result. ${ }^{8}$ Nonetheless, Al-Jamal et $\mathrm{al}^{108}$ reported one case of a long-surviving metastatic UM that was treated with BOLD and recombinant interferon. The patient had several small metastatic nodules in the liver and was not eligible for surgical resection. Following treatment, the patient was still alive after 72 months, when the report was made.

Huppert et $\mathrm{al}^{74}$ treated 48 liver metastatic UM patients with fotemustine (via the hepatic artery or via peripheral vein) and interferon- $\alpha 2$ and IL-2. Just $2 \%$ (one patient) achieved complete response, and $12.5 \%$ (six patients) achieved a partial response, with an overall response rate of $14.5 \%$.

PD-1 protein, a T-cell coinhibitory receptor, and one of its ligands, PD-L1, play a role in the capability of tumor cells to escape the host's immune system. Their blockage enhances immune function and serves as antitumor activity. Clinical trial NCT00729664 with an antibody-mediated blockade of PD-L1 induced durable tumor regression and prolonged stabilization of disease in advanced cutaneous melanoma and other types of cancer. ${ }^{111}$ Its efficacy in UM is yet to be determined.

\section{Conclusion}

UM is a frightening tumor, and despite the accuracy in the clinical diagnosis and new therapeutic modalities for treatment of the intraocular tumor, a significant proportion of the patients will develop metastasis and die of the disease. There is no difference in survival between surgical and conservative treatments of the intraocular tumor. Therefore, conservative treatments are preferred if there is a possibility to save vision. Enucleation is reserved for patients with larger tumors or with blind painful eyes, and exenteration is indicated only when there is extraocular extension and orbital invasion. Metastasis of UM can occur late in the course of the disease, and the liver is the main site of metastasis. Once patients develop metastases, the prognosis is generally poor; however, treatment of the metastatic disease does seem to improve the overall survival time. There are different modalities for the management of the 
metastatic nodules, including resection when possible, HIA chemotherapy, chemoembolization, immunoembolization, and isolated hepatic perfusion. Conventional systemic adjuvant chemotherapy does not seem to improve the overall survival of these patients, although new promising systemic therapies including TTh are gaining credibility, with several clinical trials currently in progress. Despite the different mutations found in UM and cutaneous melanoma, these tumors have been largely studied together regarding treatment options. Several studies have demonstrated that they are distinct tumors, and in our opinion they should be regarded as different entities.

\section{Disclosure}

The authors report no conflicts of interest in this work.

\section{References}

1. Singh AD, Turell ME, Topham AK. Uveal melanoma: trends in incidence, treatment, and survival. Ophthalmology. 2011;118(9):1881-1885.

2. [No authors listed]. Accuracy of diagnosis of choroidal melanomas in the Collaborative Ocular Melanoma Study. COMS report no 1. Arch Ophthalmol. 1990;108(9):1268-1273.

3. Collaborative Ocular Melanoma Study Group. The COMS randomized trial of iodine 125 brachytherapy for choroidal melanoma: V Twelve-year mortality rates and prognostic factors: COMS report no 28. Arch Ophthalmol. 2006;124(12):1684-1693.

4. McLean IW. Tumors of the Eye and Ocular Adnexa. Washington: Maryland Armed Forces Institute of Pathology; 1994.

5. Harbour JW. The genetics of uveal melanoma: an emerging framework for targeted therapy. Pigment Cell Melanoma Res. 2012;25(2):171-181.

6. Daniels AB, Lee JE, MacConaill LE, et al. High throughput mass spectrometry-based mutation profiling of primary uveal melanoma. Invest Ophthalmol Vis Sci. 2012;53(11):6991-6996.

7. Ho AL, Musi E, Ambrosini G, et al. Impact of combined mTOR and MEK inhibition in uveal melanoma is driven by tumor genotype. PLoS One. 2012;7(7):e40439.

8. Woodman SE. Metastatic uveal melanoma: biology and emerging treatments. Cancer J. 2012;18(2):148-152.

9. Buzzacco DM, Abdel-Rahman MH, Park S, Davidorf F, Olencki T, Cebulla CM. Long-term survivors with metastatic uveal melanoma. Open Ophthalmol J. 2012;6:49-53.

10. Spagnolo F, Caltabiano G, Queirolo P. Uveal melanoma. Cancer Treat Rev. 2012;38(5):549-553.

11. Callejo SA, Antecka E, Blanco PL, Edelstein C, Burnier MN Jr. Identification of circulating malignant cells and its correlation with prognostic factors and treatment in uveal melanoma. A prospective longitudinal study. Eye (Lond). 2007;21(6):752-759.

12. Blanco PL, Lim LA, Miyamoto C, Burnier MN. Uveal melanoma dormancy: an acceptable clinical endpoint? Melanoma Res. 2012;22(5):334-340.

13. De Croock L, Verbraeken H. Metastatic uveal melanoma: diagnosis and treatment. A literature review. Bull Soc Belge Ophtalmol. 2002;286:59-63.

14. Collaborative Ocular Melanoma Study Group. Assessment of metastatic disease status at death in 435 patients with large choroidal melanoma in the Collaborative Ocular Melanoma Study (COMS): COMS report no 15. Arch Ophthalmol. 2001;119(5):670-676.

15. Freton A, Chin KJ, Raut R, Tena LB, Kivelä T, Finger PT. Initial PET/CT staging for choroidal melanoma: AJCC correlation and second nonocular primaries in 333 patients. Eur J Ophthalmol. 2012; 22(2):236-243.
16. Puusaari I, Heikkonen J, Summanen P, Tarkkanen A, Kivelä T. Iodine brachytherapy as an alternative to enucleation for large uveal melanomas. Ophthalmology. 2003;110(11):2223-2234.

17. Gomer CJ. Preclinical examination of first and second generation photosensitizers used in photodynamic therapy. Photochem Photobiol. 1991;54(6):1093-1107.

18. Canal-Fontcuberta I, Salomao DR, Robertson D, et al. Clinical and histopathologic findings after photodynamic therapy of choroidal melanoma. Retina. 2012;32(5):942-948.

19. Schlötzer-Schrehardt U, Viestenz A, Naumann GO, Laqua H, Michels S, Schmidt-Erfurth U. Dose-related structural effects of photodynamic therapy on choroidal and retinal structures of human eyes. Graefes Arch Clin Exp Ophthalmol. 2002;240(9):748-757.

20. Azab M, Benchaboune M, Blinder KJ, et al. Verteporfin therapy of subfoveal choroidal neovascularization in age-related macular degeneration: meta-analysis of 2-year safety results in three randomized clinical trials: Treatment of Age-Related Macular Degeneration with Photodynamic Therapy and Verteporfin in Photodynamic Therapy Study report no 4. Retina. 2004;24(1):1-12.

21. Nowis D, Makowski M, Stokłosa T, Legat M, Issat T, Gołab J. Direct tumor damage mechanisms of photodynamic therapy. Acta Biochim Pol. 2005;52(2):339-352.

22. Barbazetto IA, Lee TC, Rollins IS, Chang S, Abramson DH. Treatment of choroidal melanoma using photodynamic therapy. Am JOphthalmol. 2003;135(6):898-899.

23. Young LH, Howard MA, Hu LK, et al. Photodynamic therapy of pigmented choroidal melanomas using a liposomal preparation of benzoporphyrin derivative. Arch Ophthalmol. 1996;114(2): 186-192.

24. Campbell WG, Pejnovic TM. Treatment of amelanotic choroidal melanoma with photodynamic therapy. Retina. 2012;32(7):1356-1362.

25. Oosterhuis JA, Journee-de Korver HG, Keunen JE. Transpupillary thermotherapy: results in 50 patients with choroidal melanoma. Arch Ophthalmol. 1998;116(2):157-162.

26. Aaberg TM Jr, Bergstrom CS, Hickner ZJ, Lynn MJ. Long-term results of primary transpupillary thermal therapy for the treatment of choroidal malignant melanoma. Br J Ophthalmol. 2008;92(6):741-746.

27. Harbour JW, Meredith TA, Thompson PA, Gordon ME. Transpupillary thermotherapy versus plaque radiotherapy for suspected choroidal melanomas. Ophthalmology. 2003;110(11):2207-2214; discussion 2215.

28. Shields CL, Shields JA, Perez N, Singh AD, Cater J. Primary transpupillary thermotherapy for small choroidal melanoma in 256 consecutive cases: outcomes and limitations. Ophthalmology. 2002;109(2):225-234.

29. Singh AD, Kivelä T, Seregard S, Robertson D, Bena JF. Primary transpupillary thermotherapy of "small" choroidal melanoma: is it safe? $\mathrm{Br}$ J Ophthalmol. 2008;92(6):727-728.

30. Shields CL, Cater J, Shields JA, et al. Combined plaque radiotherapy and transpupillary thermotherapy for choroidal melanoma: tumor control and treatment complications in 270 consecutive patients. Arch Ophthalmol. 2002;120(7):933-940.

31. Kreusel KM, Bechrakis N, Riese J, Krause L, Wachtlin J, Foerster MH. Combined brachytherapy and transpupillary thermotherapy for large choroidal melanoma: tumor regression and early complications. Graefes Arch Clin Exp Ophthalmol. 2006;244(12): 1575-1580.

32. Yarovoy AA, Magaramov DA, Bulgakova ES. The comparison of ruthenium brachytherapy and simultaneous transpupillary thermotherapy of choroidal melanoma with brachytherapy alone. Brachytherapy. 2012;11(3):224-229.

33. Caminal JM, Mejia-Castillo KA, Arias L, et al. Subthreshold transpupillary thermotherapy in management of foveal subretinal fluid in small pigmented choroidal lesions. Retina. 2013;33(1): 194-199.

34. Lichter AS, Lawrence TS. Recent advances in radiation oncology. NEngl J Med. 1995;332(6):371-379. 
35. Diener-West M, Earle JD, Fine SL, et al. The COMS randomized trial of iodine 125 brachytherapy for choroidal melanoma, II: characteristics of patients enrolled and not enrolled. COMS report no 17. Arch Ophthalmol. 2001;119(7):951-965.

36. Earle J, Kline RW, Robertson DM. Selection of iodine 125 for the Collaborative Ocular Melanoma Study. Arch Ophthalmol. 1987; 105(6):763-764.

37. Hungerford JL. Current trends in the treatment of ocular melanoma by radiotherapy. Clin Experiment Ophthalmol. 2003;31(1):8-13.

38. Wilson MW, Hungerford JL. Comparison of episcleral plaque and proton beam radiation therapy for the treatment of choroidal melanoma. Ophthalmology. 1999;106(8):1579-1587.

39. Finger PT, Chin KJ, Yu GP, Patel NS. Risk factors for cataract after palladium-103 ophthalmic plaque radiation therapy. Int J Radiat Oncol Biol Phys. 2011;80(3):800-806.

40. Finger PT, Chin KJ, Yu GP. Risk factors for radiation maculopathy after ophthalmic plaque radiation for choroidal melanoma. Am J Ophthalmol. 2010;149(4):608-615.

41. Seregard S, Damato BE, Fleming P. Uveal malignant melanoma: management options-brachytherapy. In: Saunders A, editor. Clinical Ophthalmic Oncology. Elsevier; 2007:241-247.

42. Damato B, Patel I, Campbell IR, Mayles HM, Errington RD. Local tumor control after 106Ru brachytherapy of choroidal melanoma. Int J Radiat Oncol Biol Phys. 2005;63(2):385-391.

43. Damato B, Patel I, Campbell IR, Mayles HM, Errington RD. Visual acuity after Ruthenium(106) brachytherapy of choroidal melanomas. Int J Radiat Oncol Biol Phys. 2005;63(2):392-400.

44. Sagoo MS, Shields CL, Mashayekhi A, et al. Plaque radiotherapy for juxtapapillary choroidal melanoma: tumor control in 650 consecutive cases. Ophthalmology. 2011;118(2):402-407.

45. Russo A, Laguardia M, Damato B. Eccentric ruthenium plaque radiotherapy of posterior choroidal melanoma. Graefes Arch Clin Exp Ophthalmol. 2012;250(10):1533-1540.

46. Finger PT, Chin KJ, Tena LB. A five-year study of slotted eye plaque radiation therapy for choroidal melanoma: near, touching, or surrounding the optic nerve. Ophthalmology. 2012;119(2): $415-422$.

47. Wang Z, Nabhan M, Schild SE, et al. Charged particle radiation therapy for uveal melanoma: a systematic review and meta-analysis. Int J Radiat Oncol Biol Phys. Epub October 2, 2012.

48. Char DH, Quivey JM, Castro JR, Kroll S, Phillips T. Helium ions versus iodine 125 brachytherapy in the management of uveal melanoma. A prospective, randomized, dynamically balanced trial. Ophthalmology. 1993;100(10):1547-1554.

49. Jampol LM, Moy CS, Murray TG, et al. The COMS randomized trial of iodine 125 brachytherapy for choroidal melanoma: IV. Local treatment failure and enucleation in the first 5 years after brachytherapy. COMS report no 19. Ophthalmology. 2002;109(12):2197-2206.

50. Boldt HC, Melia BM, Liu JC, Reynolds SM. I-125 brachytherapy for choroidal melanoma photographic and angiographic abnormalities: the Collaborative Ocular Melanoma Study: COMS report no 30. Ophthalmology. 2009;116(1):106-115. e1.

51. Collaborative Ocular Melanoma Study Group. Incidence of cataract and outcomes after cataract surgery in the first 5 years after iodine 125 brachytherapy in the Collaborative Ocular Melanoma Study: COMS report no 27. Ophthalmology. 2007;114(7):1363-1371.

52. Damato B, Kacperek A, Chopra M, Campbell IR, Errington RD. Proton beam radiotherapy of choroidal melanoma: the LiverpoolClatterbridge experience. Int J Radiat Oncol Biol Phys. 2005;62(5): 1405-1411.

53. Gragoudas E, Li W, Goitein M, Lane AM, Munzenrider JE, Egan KM. Evidence-based estimates of outcome in patients irradiated for intraocular melanoma. Arch Ophthalmol. 2002;120(12):1665-1671.

54. Daftari IK, Char DH, Verhey LJ, et al. Anterior segment sparing to reduce charged particle radiotherapy complications in uveal melanoma. Int J Radiat Oncol Biol Phys. 1997;39(5):997-1010.
55. Dunavoelgyi R, Dieckmann K, Gleiss A, et al. Local tumor control, visual acuity, and survival after hypofractionated stereotactic photon radiotherapy of choroidal melanoma in 212 patients treated between 1997 and 2007. Int J Radiat Oncol Biol Phys. 2011;81(1):199-205.

56. Modorati G, Miserocchi E, Galli L, et al. Gamma knife radiosurgery for uveal melanoma: 12 years of experience. Br J Ophthalmol. 2009;93(1): $40-44$.

57. Bechrakis NE, Petousis V, Willerding G, et al. Ten-year results of transscleral resection of large uveal melanomas: local tumour control and metastatic rate. Br J Ophthalmol. 2010;94(4):460-466.

58. Damato B. Progress in the management of patients with uveal melanoma. The 2012 Ashton Lecture. Eye (Lond). 2012;26(9):1157-1172.

59. Kurt RA, Gündüz K. Exoresection via partial lamellar sclerouvectomy approach for uveal tumors: a successful performance by a novice surgeon. Clin Ophthalmol. 2010;4:59-65.

60. Damato BE, Paul J, Foulds WS. Predictive factors of visual outcome after local resection of choroidal melanoma. Br J Ophthalmol. 1993; 77(10):616-623.

61. Lane AM, Egan KM, Harmon D, Holbrook A, Munzenrider JE, Gragoudas ES. Adjuvant interferon therapy for patients with uveal melanoma at high risk of metastasis. Ophthalmology. 2009;116(11): 2206-2212.

62. De Potter P, Jamart J. Adjuvant indocyanine green in transpupillary thermotherapy for choroidal melanoma. Ophthalmology. 2003;110(2): 406-413; discussion 413-414.

63. Fernandes BF, Marshall JC, Di Cesare S, Logan P, Maloney S, Burnier MN Jr. Amfenac increases the radiosensitivity of uveal melanoma cell lines. Eye (Lond). 2008;22(5):701-706.

64. Rietschel P, Panageas KS, Hanlon C, Patel A, Abramson DH, Chapman PB. Variates of survival in metastatic uveal melanoma. J Clin Oncol. 2005;23(31):8076-8080.

65. Sugihara K, Uetake H. Therapeutic strategies for hepatic metastasis of colorectal cancer: overview. J Hepatobiliary Pancreat Sci. 2012;19(5): 523-527.

66. Mariani P, Piperno-Neumann S, Servois V, et al. Surgical management of liver metastases from uveal melanoma: 16 years' experience at the Institut Curie. Eur J Surg Oncol. 2009;35(11):1192-1197.

67. Frenkel S, Nir I, Hendler K, et al. Long-term survival of uveal melanoma patients after surgery for liver metastases. $\mathrm{Br} J$ Ophthalmol. 2009;93(8):1042-1046.

68. Gündüz K, Shields JA, Shields CL, Sato T, Mastrangelo MJ. Surgical removal of solitary hepatic metastasis from choroidal melanoma. Am J Ophthalmol. 1998;125(3):407-409.

69. Aoyama T, Mastrangelo MJ, Berd D, et al. Protracted survival after resection of metastatic uveal melanoma. Cancer. 2000;89(7): $1561-1568$.

70. Collins JM. Pharmacologic rationale for regional drug delivery. J Clin Oncol. 1984;2(5):498-504.

71. Melichar B, Voboril Z, Lojik M, Krajina A. Liver metastases from uveal melanoma: clinical experience of hepatic arterial infusion of cisplatin, vinblastine and dacarbazine. Hepatogastroenterology. 2009; 56(93):1157-1162.

72. Llovet JM, Real MI, Montana X, et al. Arterial embolisation or chemoembolisation versus symptomatic treatment in patients with unresectable hepatocellular carcinoma: a randomised controlled trial. Lancet. 2002;359(9319):1734-1739.

73. Kobayashi K, Gupta S, Trent JC, et al. Hepatic artery chemoembolization for 110 gastrointestinal stromal tumors: response, survival, and prognostic factors. Cancer. 2006;107(12):2833-2841.

74. Huppert PE, Fierlbeck G, Pereira P, et al. Transarterial chemoembolization of liver metastases in patients with uveal melanoma. Eur J Radiol. 2010;74(3):e38-e44.

75. Carrasco CH, Wallace S, Charnsangavej C, Papadopoulos NE, Patt YZ, Mavligit GM. Treatment of hepatic metastases in ocular melanoma. Embolization of the hepatic artery with polyvinyl sponge and cisplatin. JAMA. 1986;255(22):3152-3154. 
76. Gonsalves CF, Eschelman DJ, Sullivan KL, Anne PR, Doyle L, Sato T. Radioembolization as salvage therapy for hepatic metastasis of uveal melanoma: a single-institution experience. AJR Am J Roentgenol. 2011;196(2):468-473.

77. Sato T, Eschelman DJ, Gonsalves CF, et al. Immunoembolization of malignant liver tumors, including uveal melanoma, using granulocyte-macrophage colony-stimulating factor. J Clin Oncol. 2008;26(33):5436-5442.

78. Yamamoto A, Chervoneva I, Sullivan KL, et al. High-dose immunoembolization: survival benefit in patients with hepatic metastases from uveal melanoma. Radiology. 2009;252(1):290-298.

79. van Iersel LB, Gelderblom H, Vahrmeijer AL, et al. Isolated hepatic melphalan perfusion of colorectal liver metastases: outcome and prognostic factors in 154 patients. Ann Oncol. 2008;19(6): 1127-1134.

80. Solanki AA, Weichselbaum RR, Appelbaum D, et al. The utility of FDG-PET for assessing outcomes in oligometastatic cancer patients treated with stereotactic body radiotherapy: a cohort study. Radiat Oncol. 2012;7(1):216.

81. Rule W, Timmerman R, Tong L, et al. Phase I dose-escalation study of stereotactic body radiotherapy in patients with hepatic metastases. Ann Surg Oncol. 2011;18(4):1081-1087.

82. Pyrhonen S. The treatment of metastatic uveal melanoma. Eur J Cancer. 1998;34 Suppl 3:S27-S30.

83. Singh AD, Bray J, Borden EC. Uveal malignant melanoma: metastasis. In: Singh AD, Damato BE, Pe'er J, Murphree AL, Perry JD, editors. Clinical Ophthalmic Oncology. Philadelphia: Saunders; 2007:283-288.

84. Kivelä T, Suciu S, Hansson J, et al. Bleomycin, vincristine, lomustine and dacarbazine (BOLD) in combination with recombinant interferon alpha-2b for metastatic uveal melanoma. Eur J Cancer. 2003;39(8): 1115-1120.

85. Albert DM, Ryan LM, Borden EC. Metastatic ocular and cutaneous melanoma: a comparison of patient characteristics and prognosis. Arch Ophthalmol. 1996;114(1):107-108.

86. Schmittel A, Schmidt-Hieber M, Martus P, et al. A randomized phase II trial of gemcitabine plus treosulfan versus treosulfan alone in patients with metastatic uveal melanoma. Ann Oncol. 2006;17(12): 1826-1869.

87. Pons F, Plana M, Caminal JM, et al. Metastatic uveal melanoma: is there a role for conventional chemotherapy? A single centre study based on 58 patients. Melanoma Res. 2011;21(3):217-222.

88. Leyvraz S, Keilholz U. Ocular melanoma: what's new? Curr Opin Oncol. 2012;24(2):162-169.

89. Schmittel A, Scheulen ME, Bechrakis NE, et al. Phase II trial of cisplatin, gemcitabine and treosulfan in patients with metastatic uveal melanoma. Melanoma Res. 2005;15(3):205-207.

90. O’Neill PA, Butt M, Eswar CV, Gillis P, Marshall E. A prospective single arm phase II study of dacarbazine and treosulfan as first-line therapy in metastatic uveal melanoma. Melanoma Res. 2006;16(3):245-248.

91. Bedikian AY, Legha SS, Mavligit G, et al. Treatment of uveal melanoma metastatic to the liver: a review of the MD Anderson Cancer Center experience and prognostic factors. Cancer. 1995;76(9): 1665-1670.

92. Flaherty LE, Unger JM, Liu PY, Mertens WC, Sondak VK. Metastatic melanoma from intraocular primary tumors: the Southwest Oncology Group experience in phase II advanced melanoma clinical trials. Am J Clin Oncol. 1998;21(6):568-572.

93. Becker JC, Terheyden P, Kämpgen E, et al. Treatment of disseminated ocular melanoma with sequential fotemustine, interferon alpha, and interleukin 2. Br J Cancer. 2002;87(8):840-845.

94. Homsi J, Bedikian AY, Papadopoulos NE, et al. Phase 2 open-label study of weekly docosahexaenoic acid-paclitaxel in patients with metastatic uveal melanoma. Melanoma Res. 2010;20(6):507-510.

95. Bedikian AY, Papadopoulos NE, Kim KB, et al. A pilot study with vincristine sulfate liposome infusion in patients with metastatic melanoma. Melanoma Res. 2008;18(6):400-404.

96. Went PT, Dirnhofer S, Bundi M, et al. Prevalence of KIT expression in human tumors. J Clin Oncol. 2004;22(22):4514-4522.
97. Pereira PR, Odashiro AN, Marshall JC, Correa ZM, Belfort R Jr, Burnier MN Jr. The role of c-kit and imatinib mesylate in uveal melanoma. J Carcinog. 2005;4:19.

98. Faingold D, Marshall JC, Antecka E, et al. Immune expression and inhibition of heat shock protein 90 in uveal melanoma. Clin Cancer Res. 2008;14(3):847-855.

99. Khalili JS, Yu X, Wang J, et al. Combination small molecule MEK and PI3K inhibition enhances uveal melanoma cell death in a mutant GNAQ- and GNA11-dependent manner. Clin Cancer Res. 2012;18(16):4345-4355.

100. Heng DY, Kollmannsberger C. Sunitinib. Recent Results Cancer Res. 2010;184:71-82.

101. Crosby MB, Yang H, Gao W, Zhang L, Grossniklaus HE. Serum vascular endothelial growth factor (VEGF) levels correlate with number and location of micrometastases in a murine model of uveal melanoma. Br J Ophthalmol. 2011;95(1):112-117.

102. Barak V, Pe'er J, Kalickman I, Frenkel S. VEGF as a biomarker for metastatic uveal melanoma in humans. Curr Eye Res. 2011;36(4): 386-390

103. Chana JS, Wilson GD, Cree IA, et al. c-myc, p53, and Bcl-2 expression and clinical outcome in uveal melanoma. Br JOphthalmol. 1999;83(1): $110-114$

104. Augsburger JJ, Corrêa ZM, Shaikh AH. Quality of evidence about effectiveness of treatments for metastatic uveal melanoma. Trans Am Ophthalmol Soc. 2008;106:128-135; discussion 135-137.

105. Kermer V, Baum V, Hornig N, Kontermann RE, Müller D. An antibody fusion protein for cancer immunotherapy mimicking IL-15 trans-presentation at the tumor site. Mol Cancer Ther. 2012;11(6): 1279-1288.

106. Bosch JJ. Immunotherapy of uveal melanoma. Dev Ophthalmol. 2012;49:137-149.

107. Danielli R, Ridolfi R, Chiarion-Sileni V, et al. Ipilimumab in pretreated patients with metastatic uveal melanoma: safety and clinical efficacy. Cancer Immunol Immunother. 2012;61(1):41-48.

108. Soni S, Lee DS, DiVito J Jr, et al. Treatment of pediatric ocular melanoma with high-dose interleukin-2 and thalidomide. J Pediatr Hematol Oncol. 2002;24(6):488-491.

109. Al-Jamal RT, Eskelin S, Pyrhonen S, Kivela T. Long-term progression-free survival in metastatic uveal melanoma after chemoimmunotherapy and consolidation thermoablation. Acta Oncol. 2009;48(3):476-479.

110. Rajpal S, Moore R, Karakousis CP. Survival in metastatic ocular melanoma. Cancer. 1983;52(2):334-336.

111. Brahmer JR, Tykodi SS, Chow LQ, et al. Safety and activity of anti-PD-L1 antibody in patients with advanced cancer. $N$ Engl J Med. 2012;366(26):2455-2465.

112. Einhorn LH, Burgess MA, Gottilieb JA. Metastatic patterns of choroidal melanoma. Cancer. 1974;34:1001-1004.

113. Gragoudas ES, Egan KM, Seddon JM, et al. Survival of patients with metastases from uveal melanoma. Ophthalmology. 1991;98(3):383-389.

114. Pyrhönen S, Hahka-Kemppinen M, Muhonen T. A promising interferon plus four-drug chemotherapy regimen for metastatic melanoma. J Clin Oncol. 1992;10(12):1919-1926.

115. Kath R, Hayungs J, Bornfeld N, et al. Prognosis and treatment of disseminated uveal melanoma. Cancer. 1993;72:2219-2223.

116. Nathan F, Sato T, Hart E. Response to combination chemotherapy of liver metastasis from choroidal melanoma compared with cutaneous melanoma. Presented at: Annual meeting of the American Society of Clinical Oncology. April 10-13, 1994; San Francisco, CA, USA.

117. Atzpodien J, Lopez Hänninen E, Kirchner H, et al. Chemoimmunotheraphy of advanced malignant melanoma: sequential administration of subcutaneous interleukin-2 and interferon-alpha after intravenous dacarbazine and carboplatin or intravenous dacarbazine, cisplatin, carmustine and tamoxifen. Eur J Cancer. 1995;31A(6): 876-881. 
118. Proebstle TM, Scheibenbogen C, Sterry W, Keilholz U. A phase II study of dacarbazine, cisplatin, interferon-alpha and high-dose interleukin-2 in "poor-risk" metastatic melanoma. Eur J Cancer. 1996;32A(9):1530-1533.

119. Nathan FE, Berd D, Sato T, et al. BOLD+interferon in the treatment of metastatic uveal melanoma: first report of active systemic therapy. J Exp Clin Cancer Res. 1997;16(2):201-208.

120. Pyrhönen S, Hahka-Kemppinen M, Muhonen T, et al. Chemoimmunotherapy with bleomycin, vincristine, lomustine, dacarbazine (BOLD), and human leukocyte interferon for metastatic uveal melanoma. Cancer. 2002;95(11):2366-2372.
121. Pföhler C, Cree IA, Ugurel S, et al. Treosulfan and gemcitabine in metastatic uveal melanoma patients: results of a multicenter feasibility study. Anticancer Drugs. 2003;14(5):337-340.

122. Bedikian AY, Papadopoulos N, Plager C, Eton O, Ring S. Phase II evaluation of temozolomide in metastatic choroidal melanoma. Melanoma Res. 2003;13(3):303-306.

\section{Publish your work in this journal}

Clinical Ophthalmology is an international, peer-reviewed journal covering all subspecialties within ophthalmology. Key topics include: Optometry; Visual science; Pharmacology and drug therapy in eye diseases; Basic Sciences; Primary and Secondary eye care; Patient Safety and Quality of Care Improvements. This journal is indexed on

\section{Dovepress}

PubMed Central and CAS, and is the official journal of The Society of Clinical Ophthalmology (SCO). The manuscript management system is completely online and includes a very quick and fair peer-review system, which is all easy to use. Visit http://www.dovepress.com/ testimonials.php to read real quotes from published authors. 